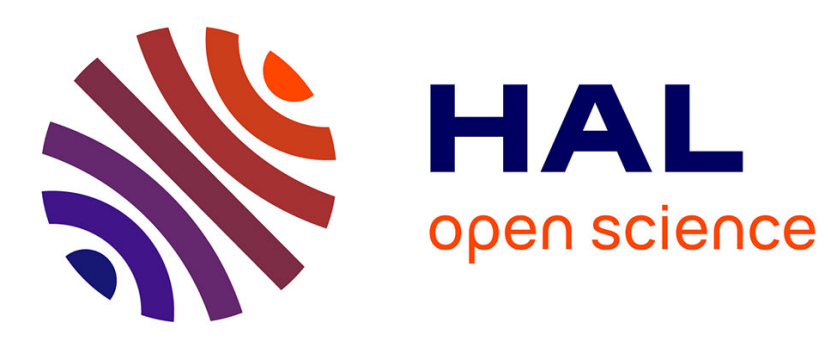

\title{
Mode I and mixed mode crack-tip fields in strain gradient plasticity \\ Stergios Goutianos
}

\section{To cite this version:}

Stergios Goutianos. Mode I and mixed mode crack-tip fields in strain gradient plasticity. International Journal of Non-Linear Mechanics, 2011, 46 (9), pp.1223. 10.1016/j.ijnonlinmec.2011.06.001 . hal00784913

\section{HAL Id: hal-00784913 \\ https://hal.science/hal-00784913}

Submitted on 5 Feb 2013

HAL is a multi-disciplinary open access archive for the deposit and dissemination of scientific research documents, whether they are published or not. The documents may come from teaching and research institutions in France or abroad, or from public or private research centers.
L'archive ouverte pluridisciplinaire HAL, est destinée au dépôt et à la diffusion de documents scientifiques de niveau recherche, publiés ou non, émanant des établissements d'enseignement et de recherche français ou étrangers, des laboratoires publics ou privés. 
Mode I and mixed mode crack-tip fields in strain gradient plasticity

Stergios Goutianos

PII:

DOI:

Reference:

To appear in:
S0020-7462(11)00146-6 doi:10.1016/j.ijnonlinmec.2011.06.001 NLM 1888

International Journal of NonLinear Mechanics

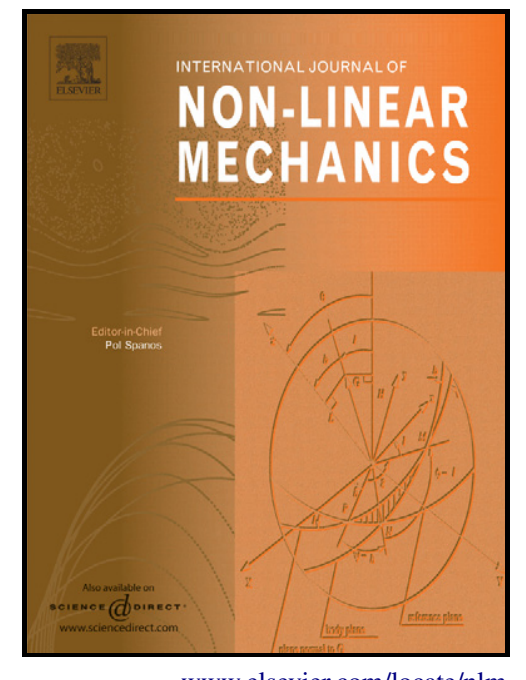

www.elsevier.com/locate/nlm

Received date: 9 October 2010

Revised date: 1 June 2011

Accepted date: 4 June 2011

Cite this article as: Stergios Goutianos, Mode I and mixed mode crack-tip fields in strain gradient plasticity, International Journal of Non-Linear Mechanics, doi:10.1016/j.ijnonlinmec.2011.06.001

This is a PDF file of an unedited manuscript that has been accepted for publication. As a service to our customers we are providing this early version of the manuscript. The manuscript will undergo copyediting, typesetting, and review of the resulting galley proof before it is published in its final citable form. Please note that during the production process errors may be discovered which could affect the content, and all legal disclaimers that apply to the journal pertain. 


\title{
Mode I and mixed mode crack-tip fields in strain gradient plasticity
}

\author{
Stergios Goutianos* \\ Materials Research Division, Risø National Laboratory for Sustainable Energy, Technical \\ University of Denmark, DK-4000 Roskilde, Denmark
}

\begin{abstract}
Strain gradients develop near the crack-tip of Mode I or mixed-mode cracks. A finite strain version of the phenomenological strain gradient plasticity theory of Fleck-Hutchinson (2001) is used here to quantify the effect of the material length scales on the crack-tip stress field for a sharp stationary crack under Mode I and mixed-mode loading. It is found that for material length scales much smaller than the scale of the deformation gradients, the predictions converge to conventional elastic-plastic solutions. For length scales sufficiently large, the predictions converge to elastic solutions. Thus, the range of length scales over which a strain gradient plasticity model is necessary is identified. The role of each of the three material length scales, incorporated in the multiple length scale theory, in altering the near-tip stress field is systematically studied in order to quantify their effect.
\end{abstract}

Keywords: plasticity; strain gradients; finite elements; crack-tip; mode mixity

\section{Introduction}

Conventional plasticity predicts that for a quasi-statically growing plane strain crack in an elastic-perfectly plastic solid in mode I, the maximum normal stress ahead of the tip is about $\underline{3}$ times the initial tensile yield stress, $\sigma_{y}[1]$.

\footnotetext{
${ }^{*}$ Current address: Saint-Gobain, High Performance Materials, Northboro-Worcester R\&D Center, 9 Goddard Road, Northboro, MA 01532, USA

Email address: stergios.goutianos@saint-gobain.com (Stergios Goutianos)
}

Preprint submitted to International Journal of Non-linear Mechanics

June 14, 2011 
Even when strain hardening is taken into account, the normal stress is less than $4-5 \sigma_{y}[2]$. The same trend also applies for mixed mode crack propagation.

Experiments on ceramic-metal interfaces have shown quasi-static crack growth of a crack along the interface in the presence of plasticity [3-6]. In these studies, the interface crack remained relatively sharp (not significant blunting) and the estimated stress level needed to result in atomistic/microscopic decohesion is about 10 times the tensile yield stress [7]. However, as pointed out above, this stress level cannot be achieved near the crack tip according to models based on conventional plasticity. This is not an issue when fracture is associated with void nucleation, growth and coalescence because the ductile fracture mechanism is of the order of $10 \mu \mathrm{m}$ or more and the crack tip stresses are about 3-5 times the tensile yield stress [8]. However, for atomistic fracture processes (observed in the experiments mentioned) conventional plasticity cannot explain the stress levels

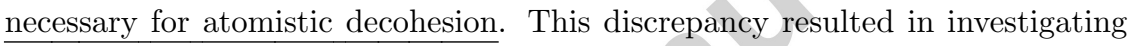
the role of the plastic strain gradients in elevating the crack-tip stresses in Mode I [7-12]. Wei and Hutchinson [13] and Wei et al. [14] have shown a large effect of the strain gradient dependent term on the crack-growth behaviour. Recently, Komaragiri et al. [15] (small strains) and Mikkelsen and Goutianos [16] (finite strains) investigated in detail the role of material length scales on crack-tip stress elevation in Mode I under plane strain using the phenomenological strain gradient plasticity theory of Fleck and Hutchinson [17].

The numerical studies of crack-tip stresses, mentioned above, use different theories of strain gradient plasticity developed over the years after the first strain gradient plasticity theory introduced by [18]. Among the number of theories existing, the more widely used are the theories due to (a) Fleck and Hutchinson [17, 19] (higher order theory) and (b) due to Nix and Gao [20] and Gao et al. [21] (lower order theory). A critical assessment of these theories was recently performed by Evans and Hutchinson [22]. It was shown that in the later theory the size effects result in an increase in hardening whereas the initial yield is almost unaffected. The former theory predicts a significant increase in yield strength and little effect on strain hardening. Another basic difference 
is that the lower order theory cannot be used to simulate passivate interfaces which require the imposition of higher order boundary conditions, i.e. plastic strain $=0$ along the interface of two different materials. In the higher order theory this is possible due to new quantities mentioned in Section 2.

With these considerations and it's computationally efficiency, the phenomenological theory of Fleck and Hutchinson [17] is used here. As in our previous work for blunted crack-tips [16], a broad range of material parameters (length scales) are systematically varied to determine the range where the gradient plasticity theory is necessary to describe the crack-tip stresses under plane strain conditions. The focus of the current work is on mixed mode crack-tip fields. However, some results for a Mode I sharp crack will be first presented.

\section{Strain Gradient Plasticity Model}

The material behaviour is modeled by a finite strain generalisation [23] of the strain gradient plasticity theory of Fleck and Hutchinson [17]. An updated Lagrangian formulation based on the work of McMeeking [24] and Yamada [25] is adopted. A comprehensive description of the reformulated strain gradient plasticity theory of Fleck and Hutchinson [17] can be found elsewhere [17, 23, 26]. Thus, only the basic features of the theory will be presented here in order to show mainly the role of the individual length scales.

A standard power-law hardening is used with the hardening modulus, $h$, and the tangent modulus, $E_{t}$ given by:

$$
\frac{1}{h}=\frac{1}{E_{t}}-\frac{1}{E}, \quad E_{t}=\frac{E}{n}\left(\frac{E E^{p}}{\sigma_{y}}+1\right)^{\frac{1}{n}-1}
$$

where $E, \sigma_{y}$, and $n$ denote the Young's modulus, the initial yield stress, and the hardening exponent of the material, respectively. In contrast to the conventional $J_{2}$ flow theory, $E_{t}$ is evaluated at $E^{p}$ (generalised effective plastic strain) and not at $\varepsilon^{p}$. The incremental form of $E^{p}$ in terms of the increment of the effective plastic strain $\left(\dot{\varepsilon}^{p}=\sqrt{3 \dot{\varepsilon}_{i j}^{p} \dot{\varepsilon}_{i j}^{p} / 2}\right)$ and its spatial derivatives, $\dot{\varepsilon}_{, i}^{p}$, is: 


$$
\dot{E}^{p^{2}}=\dot{\varepsilon}^{p^{2}}+A_{i j} \dot{\varepsilon}_{, i}^{p} \dot{\varepsilon}_{, j}^{p}+B_{, i} \dot{\varepsilon}_{, i}^{p} \dot{\varepsilon}^{p}+C \dot{\varepsilon}^{p}
$$

where tensors $A_{i j}, B_{i}$, and $C$ depend on three length material length scales, $\ell_{1}, \ell_{2}, \ell_{3}$, the outward normal to the plastic yield surface, $m_{i j}=3 S_{i j} /\left(2 \sigma_{(e)}\right)$, and its spatial derivatives, $m_{i j, k}$. The von Mises' effective stress is $\sigma_{(e)}=$ $\sqrt{3 S_{i j} S_{i j} / 2}$ where $S_{i j}=\sigma_{i j}-\frac{1}{3} \delta_{i j} \sigma_{k k}$ is the deviatoric stress, $\sigma_{i j}$ is the Cauchy stress tensor, and $\delta_{i j}$ is Kronecker's delta. A standard tensor notation is adopted where latin indices range from 1 to 3 and repeated indices denote summation. The dotted terms denote incremental quantities whereas terms with ()$_{, i}$ are partial derivatives with respect to the coordinate $x_{i}$.

The incremental principle of virtual work can be stated as [17]:

$$
\int_{V}\left(\dot{\sigma}_{i j} \delta \dot{\varepsilon}_{i j}^{e}+\dot{Q} \delta \dot{\varepsilon}^{p}+\dot{\tau}_{i} \delta \dot{\varepsilon}_{, i}^{p}\right) d V=\int_{S}\left(\dot{T}_{i} \delta u_{i}+\dot{t} \delta \dot{\varepsilon}^{p}\right) d S
$$

where $\varepsilon_{i j}^{e}$ is the elastic strain. The term $Q$ is the work conjugate to the effective plastic strain, $\varepsilon^{p}$, and its incremental form is given in Eq. 4. The higher order stress, $\tau_{i}$, is work conjugate to the gradient of the effective plastic strain, $\varepsilon_{, i}^{p}$. The traction and higher order traction on $S$ are denoted by $T_{i}$ and $t$, respectively.

$$
\dot{Q}= \begin{cases}h\left(\dot{\varepsilon}^{p}+\frac{1}{2} B_{i} \dot{\varepsilon}_{, i}^{p}+C \dot{\varepsilon}^{p}\right) & , \text { yielding } \\ \dot{\sigma}_{(e)} & , \text { elastic deformation }\end{cases}
$$

\section{Finite Element Implementation}

The above material model is implemented in a Fortran finite element code. In the finite element implementation, in addition to the nodal displacement increments, $\dot{D}^{n}$, the nodal effective plastic strain increments, $\dot{\varepsilon}_{n}^{p}$, are taken as independent unknowns. The displacement increments, $\dot{u}_{i}$, and the effective plastic strain increments, $\dot{\varepsilon}^{p}$, are then:

$$
\dot{u}_{i}=\sum_{n=1}^{m} N_{i}^{n} \dot{D}^{n}, \quad \dot{\varepsilon}^{p}=\sum_{n=1}^{l} M^{n} \dot{\varepsilon}_{n}^{p}
$$


where $N_{i}^{n}$ and $M^{n}$ are shape functions used for the incremental displacement and effective plastic strain interpolation and $m$ and $l$ are the number of nodes used in the interpolation. In the current implementation $m=8$ and $l=4$. The isoparametric geometric map from the local element coordinate system $(\xi, \eta)$ to the global coordinate system $\left(x_{1}, x_{2}\right)$ is based on $N_{i}^{n}$ shape function:

$$
x_{i}(\xi, \eta)=\sum_{n=1}^{k} N_{i}^{n}(\xi, \eta) x_{i}^{n}
$$

The element is integrated using $2 \times 2$ Gauss points. This combination is used to avoid locking in the shear dominated part near the crack-tip and spurious zero energy modes in the effective plastic strain. Different interpolation schemes were also tried using i.e. $m=l=8$ with $3 \times 3$ integration points giving significant locking in the effective plastic strain.

Using Eq. 5 in the principle of virtual work, the discretised equations can be obtained [23]:

$$
\left[\begin{array}{ll}
\mathbf{K}_{\mathbf{e}} & \mathbf{K}_{\mathbf{e p}} \\
\mathbf{K}_{\mathbf{e p}}^{\mathbf{T}} & \mathbf{K}_{\mathbf{p}}
\end{array}\right]\left[\begin{array}{c}
\dot{D} \\
\dot{\varepsilon}^{p}
\end{array}\right]=\left[\begin{array}{c}
\dot{\mathbf{F}}_{\mathbf{1}} \\
\dot{\mathbf{F}}_{\mathbf{2}}
\end{array}\right]+\left[\begin{array}{c}
\mathbf{C}_{\mathbf{1}} \\
\mathbf{C}_{\mathbf{2}}
\end{array}\right]
$$

where $\mathbf{K}_{\mathbf{e}}$ is the elastic stiffness matrix, $\mathbf{K}_{\mathbf{e p}}$ is a matrix of dimension force and $\mathbf{K}_{\mathbf{p}}$ is a matrix of dimension energy. The conventional external incremental force vector is $\dot{\mathbf{F}}_{\mathbf{1}}$ and $\dot{\mathbf{F}}_{\mathbf{2}}$ is the incremental higher order force vector. The second term in the right-hand side represents the equilibrium correction vector where $\mathbf{C}_{\mathbf{1}}$ is the standard force equilibrium term and $\mathbf{C}_{\mathbf{2}}$ is the equilibrium term related to the increments of the effective plastic strain. In the current implementation $\mathbf{C}_{\mathbf{2}} \equiv 0$. The expressions for all terms can be found in [23].

The solution of Eq. 7 gives the nodal displacement and effective plastic strain increments. From these incremental values the updated strains, $\varepsilon_{i j}$, stresses, $\sigma_{i j}$, higher order stresses, $\tau_{i}$, and $Q$ are computed at each integration point. If yielding occurs at an integration point then the effective plastic strain, $E^{p}$, is updated. The yielding criterion for an integration point is $Q>Q_{\max }$ where $Q_{\max }$ is the maximum value of $Q$ attained in the integration point. The initial value 
of $Q$ is $\sigma_{y}$. Elastic unloading in an integration point occurs when $\dot{\varepsilon}^{p}<0$. In the elastic region a sufficiently large hardening modulus, $h_{\text {elastic }}=10^{5} \mathrm{E}$, is introduced to avoid spurious effective plastic strain increments. This corresponds to an internal elastic/elastic-plastic boundary condition $\dot{\varepsilon}^{p}=0$ [27].

\section{Model}

A semi-infinite crack is modeled a) in a homogeneous elastic-plastic solid under plane strain conditions for Mode I simulations, and b) along the interface of an elastic-plastic solid and a rigid solid for mixed mode computations. Thus, in both cases only the upper half plane is modeled as can be seen in Fig. 1a with appropriate boundary conditions as explained later in this section.

For finite deformation analysis the crack is assigned a finite curvature. More specifically, the crack-tip is an ellipse with semi-axes $\alpha$ and $\gamma$ (see Fig. 1b). In the boundary layer analysis the ratio $\gamma / \alpha$ is 0.05 and $\alpha$ is equal to $10^{-4}$ times $R$, where $R$ is the distance from the crack-tip to boundary at which the tractions are applied. For loadings causing severe crack-tip blunting the solution does not depend on the initial crack geometry (a number of ratios $\gamma / \alpha$ ranging from 1.0 to 0.01 were initially tried). The obtained solutions, thus, may be interpreted as those corresponding to an initially sharp crack. An illustration of the mesh near the crack-tip is given in Fig. 1c to show the mesh density.

\subsection{Boundary Conditions}

For Mode I simulations, symmetry displacement boundary conditions are applied along the symmetry line $\left(0 \leq x_{1} \leq R, x_{2}=0\right)$ in front of the crack-tip (Fig. 1a). Along the same boundary, no restriction is imposed on $\epsilon^{p}$ which implies that the higher order stresses are equal to zero along this line. In mixed mode computations, $\epsilon^{p}=0$ along the line in front of the crack-tip assuming that dislocation motion is blocked by the rigid solid (passivated surface). 


\subsubsection{Mode $I$}

In Mode I, a $K_{I}$ field is prescribed through displacements in the x- and y-directions at distance $R$ from the crack-tip:

$$
\left\{\begin{array}{l}
u_{1} \\
u_{2}
\end{array}\right\}=\frac{K_{I}}{2 G} \sqrt{\frac{R}{2 \pi}}\left\{\begin{array}{c}
\cos \theta / 2\left[k-1+2 \sin ^{2} \theta / 2\right] \\
\sin \theta / 2\left[k+1-2 \cos ^{2} \theta / 2\right]
\end{array}\right\}
$$

where $G$ denotes the shear modulus of the material and $k=3-4 \nu$ for plane strain, and $\nu$ is the Poisson's ratio.

\subsubsection{Mixed Mode}

For mixed mode small scale yielding, the tractions along the interface are given by two stress intensity factor components $K_{1}$ and $K_{2}$ :

$$
\sigma_{22}+i \sigma_{12}=\left(K_{1}+i K_{2}\right)(2 \pi r)^{-1 / 2} r^{-i \epsilon}
$$

where $r$ is the distance from the crack-tip and $\epsilon$ is the oscillation index:

$$
\epsilon=\frac{1}{2 \pi} \ln \left(\frac{1-\beta}{1+\beta}\right)
$$

and $\beta$ is the second Dundur's parameter

$$
\beta=\frac{1}{2} \frac{\mu\left(1-2 \nu_{s}\right)-\mu_{s}(1-2 \nu)}{\mu\left(1-\nu_{s}\right)+\mu_{s}(1-\nu)}
$$

with $\mu=E /(2(1+\nu))$ and $\mu_{s}=E_{s} /\left(2\left(1+\nu_{s}\right)\right) . E_{s}=10^{3} E$ and $\nu_{s}=0.3$ are the Young's modulus and Poisson's ratio of the rigid solid, respectively. The magnitude of the stress intensity factors is:

$$
|K|=\sqrt{K_{1}^{2}+K_{2}^{2}}
$$

If $L$ is a reference length characterising the remote field, then a mode mixity measure, $\psi$, which depends on $\mathrm{L}$ is:

$$
\tan \psi=\frac{\operatorname{Im}\left[K_{1}+i K_{2}\right] L^{i \epsilon}}{\operatorname{Re}\left[K_{1}+i K_{2}\right] L^{i \epsilon}}
$$


From Eqs. 9 and 13 it can be seen that $L$ measures the relative ratio of shear to normal stress at distance $r=L$ on the interface [28] according to the elastic solution. The displacement field for the singular field in the elastic-plastic solid is then:

$$
\begin{aligned}
& \mu\left(u_{i}+i u_{2}\right)=\frac{|K| r^{1 / 2}}{2 \sqrt{2 \pi} \cosh (\pi \epsilon)} \\
& \left\{\frac{k e^{i \theta / 2+\epsilon(\theta-\pi)-i \widetilde{\psi}}}{1-2 i \epsilon}-\frac{\epsilon^{-i \theta / 2-\epsilon(\theta-\pi)-i \widetilde{\psi}}}{1-2 i \epsilon}-i \sin \theta e^{i \theta / 2+\epsilon(\theta-\pi)+i \widetilde{\psi}}\right\}
\end{aligned}
$$

where the $\widetilde{\psi}$ dependence on $r$ is given by:

$$
\widetilde{\psi}=\psi+\epsilon \ln (r / L)
$$

In the simulations the displacements $u_{1}$ and $u_{2}$ are controlled at the boundary $(r=R)$ through Eq. 14. Similar to Tvergaard and Hutchinson [28] an alternative measure of mode mixity, $\psi_{p}$, is defined based on reference length, $R_{p}$, which gives the approximate plastic zone size. The relation between $\psi$ and $\psi_{p}$ is given by:

$$
\psi_{p}=\psi+\epsilon \ln \left(R_{p} / L\right)
$$

where the plastic zone size, $R_{p}$, is:

$$
R_{p}=\frac{1}{3 \pi}\left(\frac{|K|}{\sigma_{y}}\right)^{2}
$$

The applied loading is, thus, given by $R_{p}$ (through $|K|$ ), $\psi$ and $L$. For the case of Mode I, $R_{p}$ is defined by Eq. 17 with $|K|$ replaced by $K_{I}$. The phase angle, $\psi_{p}$ in Eq. 16 is a measure of the near-tip mode mixity [29].

\section{Results and Discussion}

\subsection{Mode I}

As mentioned the Mode I case has been studied in great detail by Komaragiri et al. [15] (small strains - sharp crack) and Mikkelsen and Goutianos [16] (finite 
strains - blunted crack). The mean stress $\left(\sigma_{m}=\left(\sigma_{11}+\sigma_{22}+\sigma_{33}\right) / 3\right)$ at a fixed distance equal to $0.1 \% R_{p}$ and $\theta=0^{\circ}([15])$ is plotted in Fig. 2 as function of the material length scales $\left(\ell_{1}=\ell_{2}=\ell_{3}=\ell\right.$ ) for various strain hardening exponents, $n$. Three distinct regions can be identified: a) for small length scale, $\ell$, the solutions converge to conventional plasticity predictions which strongly depend on the strain hardening exponent [30], b) for large $\ell$ the solutions approach the elastic solution which of course is independent of $n$ and c) in between these two regions is the area where strain gradient plasticity has a profound effect (approximately for $10^{-3} \leq \ell / R_{p} \leq 1$, depending on $n$ ) in elevating the mean stress. The same trend has been shown for $\sigma_{\theta \theta},\left(\theta=0^{\circ}\right)$ by [16].

In addition to the mean stress ahead of the crack-tip, two additional measures are introduced to compare the effect of the material length scales on the crack-tip fields. The first one is the conventional plastic energy [17]:

$$
Q_{\varepsilon^{p}}=\int_{v} \int_{0}^{\varepsilon^{p}} \sigma_{(e)}\left(\tilde{\varepsilon}^{p}\right) d \tilde{\varepsilon}^{p} d V=\int_{v} \int_{0}^{\varepsilon^{p}} \sigma_{i j}\left(\tilde{\varepsilon}_{i j}^{p}\right) d \tilde{\varepsilon}_{i j}^{p} d V
$$

In the present work, the conventional plastic energy was preferred over the $\underline{\text { higher order plastic energy. The theory of Fleck and Hutchinson [17], used here, }}$ does not guarantee higher order positive plastic work [31, 32]. Thus, although the (total) higher order plastic energy was always found positive in the calcu$\underline{\text { lations, the conventional plastic energy, which is ensured to be positive, is pre- }}$ ferred. The second measure is the commonly used crack opening displacement, $\delta$, defined in Fig. $1 \mathrm{~b}[33,34]$. It should be noted that $\delta$ is defined here as half the crack opening for Mode I in contrast to the standard definition of total crack opening. Since the same measure is used later for the mixed mode cases the definition of Fig. 1b is preferred for the purposes of the current work.

The normalised conventional plastic energy as a function of the material length scales for the same material properties and loading as in Fig. 2 is displayed in Fig. 3. The corresponding graph for the crack opening displacement is given in Fig 4. The same trend as in Fig. 2 can be seen. There is a smooth transition from the $J 2$ conventional plasticity solution to the elastic solution 
and this transition occurs approximately for $10^{-3} \leq \ell / R_{p} \leq 1$. It should be emphasised that although the stress field approaches the elastic solution for large length scales as shown in Fig. 2, that it does not mean that the material is not yielding [16]. The gradient dependent terms, which result in the stress elevation $\underline{\text { near the crack tip, are active only in the presence of plastic strain gradients. }}$ Thus, for large material length scales, the plastic deformation at the crack tip is $\underline{\text { suppressed (see Fig. } 3 \text { ) by the gradient dependent terms resulting in an essential }}$ linear elastic response. In Fig. 3 the conventional plastic energy, $Q_{\varepsilon^{p}}$, is normalised with the strain energy $\Phi_{H R R}\left(R_{p}\right)$ within a semi-circular region of radius $R_{p}$ centred at the crack-tip in case of Hutchinson-Rice-Rosengren (HRR) singular plane strain field in a power law material described by a Ramberg-Osgood curve $[35]$ :

$\Phi_{H R R}\left(R_{p}\right)=\frac{1}{2}\left(\frac{S_{n}}{I_{n}}\right) J R_{p}=\frac{1}{2}\left(\frac{S_{n}}{I_{n}}\right) \frac{\left(1-\nu^{2}\right) K_{I}^{2}}{E} R_{p}=\frac{3 \pi}{2}\left(\frac{S_{n}}{I_{n}}\right)\left(\frac{1-\nu^{2}}{E}\right) \sigma_{y}^{2} R_{p}^{2}$

where the dimensionless constants $S_{n}$ and $I_{n}$ depend only on $n$, and $J$ is the J-integral. Similarly, the crack opening displacement, $\delta$, is normalised with half the crack-tip opening displacement $2 \delta_{H R R}$ given by:

$$
\delta_{H R R}=\frac{1}{2}\left(\frac{\alpha \sigma_{y}}{E}\right)^{\frac{1}{n}} D_{n} \frac{J}{\sigma_{y}}=\frac{3 \pi}{2}\left(\frac{\alpha \sigma_{y}}{E}\right)^{\frac{1}{n}} D_{n}\left(\frac{1-\nu^{2}}{E}\right) \sigma_{y} R_{p}
$$

where the dimensionless constants $D_{n}$ depends only on $n$.

A number of experimental techniques have been used to measure the length scale parameters such as nanoindentation [36], torsion [37] and microbend [38]. Alternatively, it is envisaged that the material length scales can be inferred by comparing numerical results such as of Fig. 4 with experimental near crack-tip opening profiles using techniques as in [39].

Next in Fig. 5 the role of the individual length scale parameters in suppressing the plastic deformation at the crack-tip is examined for a fixed strain hardening exponent $(n=5)$. It can be seen that for a given length scale $\ell$ (in the range where $\ell$ has an effect) the case $\ell=\ell_{2}$ gives a smaller effect (increased 
conventional plastic energy) than $\ell=\ell_{3}$. It is interesting to observe that for $\ell / R_{p}<0.04$ the case $\ell=\ell_{1}$ suppresses plastic deformation more the $\ell=\ell_{2}$. With increasing $\ell / R_{p}$ the difference decreases and for $\ell / R_{p}>1$ the case $\ell=\ell_{1}$ is almost identical to the $\ell=\ell_{3}$. The case where all the length scales are equal to each other, $\ell_{1}=\ell_{2}=\ell_{3}=\ell$, displays the largest strain gradient effects than the other three cases where only one length scale is present.

Fig. 6 shows the role of the individual length scales in the crack-tip opening. For $\ell / R_{p}<0.02$ the case $\ell=\ell_{3}$ displays smaller strain gradient effects than the $\ell=\ell_{2}$ and this trend reverses for larger $\ell / R_{p}$ values. The case $\ell_{1}=\ell_{2}=$ $\ell_{3}=\ell$ results in smaller crack-tip openings than the case of individual length scales except the region for $\ell / R_{p}<0.01$ when compared to $\ell=\ell_{2}$ and $\ell=\ell_{3}$. The case $\ell=\ell_{1}$ displays the most unexpected behaviour in the area around $\ell / R_{p}=0.01$ in the sense that the resulted crack-tip openings are larger than the opening corresponding to the conventional plasticity solution. This behaviour is further examined in Fig. 7, where the shape of the deformed crack is plotted for $\ell / R_{p}=0.01$. It can be seen that the crack opening for $\ell=\ell_{1}$ is larger than the openings for the other three cases in general and not only at a single point (the intersection with the $45^{\circ}$ line) which is used in Fig. 6.

As demonstrated above and in the previous works $[15,16]$, the large plastic deformation gradients in the crack-tip region increase the amount of plastic flow resistance and hence lower the amount of plastic deformation in this region. This is shown in more details in Figs. 8 and 9. Fig. 8 shows the normalised stress, $\sigma_{m} / \sigma_{y}$, along the symmetry line in front of the crack-tip (see Fig. 1c) for two $\ell / R_{p}$ ratios and four different material length scale combinations. The $\ell / R_{p}=0.01$ ratio corresponds to the case where the strain gradient dependent terms start to influence the stress field around the crack-tip (see Fig. 5). As $\ell / R_{p}$ increases to 0.25 the strain gradient terms dominate and prevent plastic deformation to develop (Fig. 5) and therefore the stresses in front of the cracktip approach the elastic solution. 


\subsection{Mixed Mode}

The results for the conventional plastic energy as a function of the mode mixity, $\psi_{p}$, for various material length scales (for $\ell_{1}=\ell_{2}=\ell_{3}=\ell$ ) are presented in Fig. 11. The elastic-plastic solid / rigid solid interface is modeled as a passivated layer where the plastic strain vanishes [22]. Previous numerical and experimental studies using conventional plasticity theory (i.e. [28, 40, 41]) have demonstrated the strong mixed mode effect on toughness when plastic deformation occurs at the crack-tip in at least one of the materials joined across the interface. The same effect can be seen also in Fig. 11 for low $\ell / R_{p}$ values. Based on the results of the previous section as $\ell / R_{p}$ increases the plastic deformation at the crack-tip is suppressed for all mode mixities. The minima of the curves of Fig. 11 fall near $\psi_{p}=0^{\circ}$ indicating that $\psi_{p}$ is a local measure.

The effect of the material length scales for the various mode mixities is more clearly shown in Fig. 12 where the normalised conventional plastic energies of Fig. 11 are divided with the normalised conventional plastic energy for $\ell / R_{p}=0.00075$ (which approach the conventional plasticity solution) and each $\psi_{p}$. The most obvious observation is that the effect of the material length scales in suppressing the plastic deformation increases as $\psi_{p} \rightarrow 0^{\circ}$.

Boundaries of the plastic zones are shown in Fig. 13 for three values of $\psi_{p}$ and three $\ell / R_{p}$ ratios for each $\psi_{p}$. Similar to Fig. 10, an increase of $\ell / R_{p}$ results in a decrease of the plastic zone length ahead of the crack-tip $\left(x_{1} \geq 0\right.$ and $x_{2}=0$ ) but the overall change in the plastic zone boundaries is small.

Fig. 14 depicts the contours of generalised effective plastic strain, $E^{p}$, that correspond to Fig. $13 \mathrm{~b}$ for $\ell / R_{p}=0.01$, and 1.0 , respectively. As it was the case for pure Mode I (see Fig. 9) a large incorporated length scale results in large plastic deformation gradients in the crack-tip region, resulting in larger gradient dependent generalised effective plastic strains, $E^{p}$. This increases the plastic flow resistance which result in smaller plastic deformation in this region as can also be seen in Fig. 11.

Fig. 15 shows the mean stress, at a fixed distance equal to $0.1 \% R_{p}$ and $\theta=0^{\circ}$ as in Fig. 2, for various length scales $\ell / R_{p}$ with $\ell_{1}=\ell_{2}=\ell_{3}=\ell$ and 
mode mixities $\psi_{p}$. It is interesting to observe that the peak stress for each $\psi_{p}$ is maximum at an intermediate $\ell / R_{p}$ ratio and then decreases to reach a plateau value for large $\ell / R_{p}$ ratios. The reason for this unexpected behaviour is not clear at the moment. In contrast to the results of Fig. 11, Fig. 15 depicts a single value for the $\sigma_{m}$ at a certain distance from the crack-tip. To further investigate the dependence of $\sigma_{m}$ on $\ell / R_{p}$, Fig. 16 shows the mean stress as function of the distance from the crack-tip. Although the peak stress (at $0.1 \%$ of $R_{p}$ ) is higher for a lower $\ell / R_{p}$ ratio, the overall stress level increases with increasing $\ell / R_{p}$ in accordance with Fig. 11. Thus, it can be argued that the conventional plastic energy (11) is in this case a better measure of the effect of the length scales than the peak (mean) stress based on a single value.

Next in Figs. 17, 18 and 19 the effect of the length scales on the plastic energy, crack opening displacement and mean stress are presented for the cases where only one of the three length scales is non-vanishing. As in the previous section, the case $\ell_{1}=\ell_{2}=\ell_{3}=\ell$ is also included. The conventional plastic energy (see Fig. 17) is smaller when all the length scales are non-vanishing for both $\ell / R_{p}$ ratios shown. Under mix mode conditions the length scales $\ell_{2}$ and $\ell_{3}$, related to rotational gradients, introduce additional strain hardening. The length scales $\ell_{2}$ and $\ell_{3}$ have approximately the same effect in suppressing the plastic deformation at the crack-tip, whereas the case $\ell_{1}=\ell$ results in the large accumulated conventional plastic energy.

The crack opening displacements are shown in Fig. 18. As with the plastic conventional energy, the crack opening displacement, for each $\psi_{p}$, is smaller for $\ell_{1}=\ell_{2}=\ell_{3}=\ell$ than when only one length scale is non-vanishing. It is interesting to observe that for $\psi_{p}=-30^{\circ}$, the crack opening is smaller when $\ell_{1}=\ell$ than for the other two cases, $\ell_{2}=\ell$ or $\ell_{3}=\ell$. The same trend is observed for $\psi_{p}=0^{\circ}$. However, for $\psi_{p}=-30^{\circ}$ the differences between the three cases are very small.

Fig. 19 depicts the mean stress calculated, as above, at a distance equal to $0.1 \% R_{p}$ from the crack-tip for different $\ell / R_{p}$ ratios and three different mode mixities $\psi_{p}$. The focus is for $\ell / R_{p}$ ratios larger than 0.01 , since it is for these 
values that strain gradients have a strong influence on the stress field. For

$\psi_{p}=30^{\circ}$, it can be seen that $\sigma_{m}^{\ell_{1}=\ell_{2}=\ell_{3}=\ell}>\sigma_{m}^{\ell_{1}=\ell}>\sigma_{m}^{\ell_{2}=\ell}>\sigma_{m}^{\ell_{3}=\ell}$ until $\ell / R_{p} \approx 0.1$. The same trend is observed also for $\psi_{p}$ equal to $0^{\circ}$ and $-30^{\circ}$ but the transition point is different.

\section{Concluding remarks}

A broad parametric study has been performed to identify the relationship between the material length scales and the plastic deformation at the crack-tip of Mode I and mixed mode stationary cracks. The main findings are:

- In all cases (Mode I and mixed mode cracks) it was found that when the material length scales are very small compared to the plastic zone size (approximately $\ell / R_{p}<0.001$ ) then the solution coincides with the conventional $J 2$ plasticity theory. For large material length scales (approximately $\ell / R_{p}>1$ ) the predictions coincide with the elastic solutions. It is in the intermediate range, $0.001<\ell / R_{p}<1$, where a strain gradient plasticity theory is necessary.

- The size of the yielding region ahead of the crack-tip $\left(\theta=0^{\circ}\right)$ decreases as the material length scales are becoming large compared to the plastic zone. However, the overall plastic zone shape is not significantly changed. The large differences observed for Mode I and mixed mode cracks as $\ell / R_{p}$ increases are mainly due to the magnitude of the plastic deformation gradients.

- For both Mode I and mixed mode cracks when all the material length scales are included $\left(\ell_{i} \neq 0, \mathrm{i}=1,2,3\right)$ the strain gradient effects are significantly higher than when only one material length scale is included.

- Similar to J2 plasticity, as the mode mixity increases, the plastic energy is also increasing for fixed material length scales. The effect of the strain gradients is larger for small mode mixities $\left(\psi_{p} \rightarrow 0^{\circ}\right)$. 


\section{References}

[1] W. J. Drugan, J. R. Rice, L. Sham, T., Asymptotic analysis of growing plane strain tensile cracks in elastic-ideally plastic solids, J. Mech. Phys. Solids 30 (1982) 447-473.

[2] V. Tvergaard, J. W. Hutchinson, The relation between crack growth resistance and fracture process parameters in elastic-plastic solids, J. Mech. Phys. Solids 40 (1992) 1377-1397.

[3] G. Elssner, D. Korn, M. Ruhle, The influence of interface impurities on fracture energy of uhv diffusion bonded metal-ceramic bicrystals, Scripta Metall. Mater 31 (1994) 1037-1042.

[4] D. Korn, G. Elssner, R. M. Cannon, M. Ruhle, Fracture properties of interfacially doped nb-a12o3 bicrystals: I, fracture characteristics, Acta Mater 50 (2002) 3881-3901.

[5] A. Bagchi, G. E. Lucas, Z. Suo, A. G. Evans, A new procedure for measuring the decohesion energy of thin ductile films on substrates, J. Mater. Res. 9 (1994) 1744-1741.

[6] A. Bagchi, A. G. Evans, The mechanics and physics of thin film decohesion and its measurement, Interface Sci. 3 (1996) 169-193.

[7] J. Y. Chen, Y. Wei, Y. Huang, J. W. Hutchinson, K. C. Hwang, The crack tip fields in strain gradient plasticity: the asymptotic and numerical analyses, Eng. Fract. Mech. 64 (1999) 625-648.

[8] Z. C. Xia, J. W. Hutchinson, Crack tip fields in strain gradient plasticity, J. Mech. Phys. Solids 44 (1996) 1621-1648.

[9] S. Qu, Y. Huang, H. Jiang, C. Liu, P. D. Wu, K. C. Hwang, Fracture analysis in the conventional theory of mechanism-based strain gradient (cmsg) plasticity, Int. J. Fract. 129 (2004) 199-220. 
[10] S. H. Chen, T. C. Wang, Finite element solutions for plane strain mode i crack with strain gradient effects, Int. J. Solids Struct. 39 (2002) 1241-1257.

[11] K. C. Hwang, H. Jiang, Y. Huang, H. Gao, Finite deformation analysis of mechanism-based strain gradient plasticity: torsion and crack tip field, Int. J. Plast. 19 (2003) 235-251.

[12] H. Jiang, Y. Huang, Z. Zhuang, K. C. Hwang, Fracture in mechanism-based strain gradient plasticity, J. Mech. Phys. Solids 49 (2001) 979-993.

[13] Y. Wei, J. Hutchinson, Steady-state crack growth and work of fracture for solids characterized by strain gradient plasticity, J. Mech. Phys. Solids 45 (1997) 1253-1273.

[14] Y. Wei, X. Qui, K. C. Hwang, Steady-state crack growth and fracture work based on the theory of mechanism-based strain gradient plasticity, Eng. Fract. Mech. 71 (2004) 107-125.

[15] U. Komaragiri, S. R. Agnew, R. P. Gangloff, M. R. Begley, The role of macroscopic hardening and individual length-scales on crack tip stress elevation from phenomenological strain gradient plasticity, J. Mech. Phys. Solids 56 (2008) 3527-3540.

[16] L. P. Mikkelsen, S. Goutianos, Suppressed plastic deformation at blunt crack tips due to strain gradient effects, Int. J. Solids Struct. 46 (2009) $4430-4436$.

[17] N. A. Fleck, J. W. Hutchinson, A reformulation of strain gradient plasticity, J. Mech. Phys. Solids 49 (2001) 2245-2271.

[18] E. C. Aifantis, On the microstructural origin of certain inelastic modes, Trans. ASME J. Eng. Mat. Tech. 106 (1984) 326-330.

[19] N. A. Fleck, J. W. Hutchinson, Strain gradient plasticity, Adv. Appl. Mech. 37 (1997) 295-361. 
[20] W. D. Nix, H. J. Gao, Indentation size effects in crystalline materials: a law for strain gradient plasticity, J. Mech. Phys. Solids 46 (1998) 411-425.

[21] H. Gao, Y. Huang, W. D. Nix, J. W. Hutchinson, Mechanism-based strain gradient plasticity -i. theory, J. Mech. Phys. Solids 47 (1999) 1239-1263.

[22] A. G. Evans, J. W. Hutchinson, A critical assessment of theories of strain gradient plasticity, Acta Mater. 57 (2009) 1675-1688.

[23] C. F. Niordson, P. Redanz, Size-effects in plane strain sheet-necking, J. Mech. Phys. Solids 52 (2004) 2431-2454.

[24] R. M. McMeeking, J. R. Rice, Finite-element formulations for problems of large elastic-plastic deformation, Int. J. Solids Struct. 11 (1975) 601-616.

[25] Y. Yamada, M. Sasaki, Elastic-plastic large deformation analysis program and lamina compression test, Int. J. Mech. Sci. 37 (1995) 691-707.

[26] C. F. Niordson, J. W. Hutchinson, Non-uniform plastic deformation of micron scale objects, Int. J, Numer. Meth. Engng. 56 (2003) 961-975.

[27] C. F. Niordson, On higher-order boundary conditions at elastic-plastic boundaries in strain-gradient plasticity, Philosophical Magazine 88 (2008) 3731-3745.

[28] V. Tvergaard, J. W. Hutchinson, The influence of plasticity on mixed mode interface toughness, J. Mech. Phys. Solids 41 (1993) 1119-1135.

[29] Y. Wei, J. W. Hutchinson, Toughness of ni/al203 interfaces as dependent on micron-scale plasticity and atomistic-scale separation, Philosophical Magazine 88 (2009) 3841-3859.

[30] R. M. McMeeking, Finite deformation analysis of crack-tip opening in elastic-plastic materials and implications for fracture, J. Mech. Phys. Solids 25 (1977) 357-381. 
[31] P. Gudmundson, A unified treatment of strain gradient plasticity, J. Mech. Phys. Solids 52 (2004) 1379-1406.

[32] M. E. Gurtin, L. Anand, Thermodynamics applied to gradient theories involving the accumulated plastic strain: The theories of aifantis and fleck and hutchinson and their generalization., J. Mech. Phys. Solids 57 (2009) $405-421$.

[33] C. F. Shih, Relationship between the j-integral and the crack opening displacement for staionary and extending cracks, J. Mech. Phys. Solids 29 (1981) 305-326.

[34] N. p. O'Dowd, C. F. Shih, Family of crack-tip fields characterized by a triaxility parameter -i. structure of fields, J. Mech. Phys. Solids 39 (1991) 989-1015.

[35] C. F. Shih, Tables of Hutchinson-Rice-Rosengren singular field quantities, Technical Report, Division of Engineering, Brown University, 1983.

[36] W. J. Poole, M. F. Ashby, N. A. Fleck, Micro-hardness of annealed and workhardened copper polycrystals, Scripta Mater. 34 (1996) 559-564.

[37] N. A. Fleck, G. M. Muller, M. F. Ashby, J. W. Hutchinson, Strain gradient plasticity: theory and experiment, Acta Metall. Mater. 42 (1994) 475-487.

[38] J. S. Stölken, A. G. Evans, A microbend test method for measuring the plasticity length scale, Acta Mater. 46 (1998) 5109-5115.

[39] B. F. Sørensen, A. Horsewell, O. Jorgensen, A. N. Kumar, Fracture resistance measurement method for in situ observation of crack mechanisms, J. Am. Ceram. Soc. 81 (1998) 661-669.

[40] K. M. Liechti, Y. S. Chai, Asymmetric shielding in interfacial fracture under in-plane shear, J. Appl. Mech. 59 (1992) 296-304.

[41] K. M. Liechti, A. W. Mello, The effect of self-assembled monolayers on interfacial fracture, J. Appl. Mech. 73 (2006) 860-870. 


\section{Figure captions:}

Figure 1: (a) Finite element mesh for the semi-infinite crack geometry , (b) schematic of the crack-tip geometry, and (c) near-tip mesh details.

Figure 2: Mean stress at a distance equal to $0.1 \% R_{p}$ from the crack-tip $\left(\theta=0^{\circ}\right)$ as function of the material length scales for various strain hardening exponents.

Figure 3: Normalised conventional plastic energy as function of the material length scales for various strain hardening exponents.

Figure 4: Normalised crack opening displacement as function of the material length scales for various strain hardening exponents.

Figure 5: Normalised conventional plastic energy for various material length scales $(n=5)$.

Figure 6: Normalised crack opening displacement for various material length scales $(n=5)$.

Figure 7: Crack opening profile $(n=5)$.

Figure 8: Mean stress, $\sigma_{m}$, along the symmetry line in front of the crack-tip.

Figure 9: Contours of generalised effective plastic strain, $E^{p}$, for (a) $\ell / R_{p}=$ 0.005 , and (b) $\ell / R_{p}=0.25\left(\ell_{1}=\ell_{2}=\ell_{3}=\ell\right)$.

Figure 10: Small-scale yielding boundaries of the plastic zone for three different $\ell_{1}\left(\ell_{2}=\ell_{3}=0\right)$.

Figure 11: Crack tip normalised conventional plastic energy versus the local mixity measure $\psi_{p}$.

Figure 12: Normalised conventional plastic energy over the normalised $Q_{\varepsilon^{p}}$, for $\lambda=\ell / R_{p}=0.00075$, versus the local mixity measure $\psi_{p}$. The normalisation of $Q_{\varepsilon^{p}}$ is given in Fig. 11 .

Figure 13: Boundaries of the plastic zone. The mode mixity is (a) $\psi_{p}=$ $-45^{\circ}$, (b) $\psi_{p}=0^{\circ}$, and (c) $\psi_{p}=+45^{\circ}$.

Figure 14: Contours of generalised effective plastic strain, $E^{p}$, for (a) $\ell / R_{p}=0.01$, and (b) $\ell / R_{p}=1.0\left(\psi_{p}=0^{\circ}\right.$ and $\left.\ell_{1}=\ell_{2}=\ell_{3}=\ell\right)$. 
Figure 15: Mean stress at a distance equal to $0.1 \% R_{p}$ from the crack-tip $\left(\theta=0^{\circ}\right)$ as a function of the material length scales for various mode mixities $\psi_{p}$.

Figure 16: Mean stress, $\sigma_{m}$, along the interface in front of the crack-tip.

Figure 17: Crack tip normalised conventional plastic energy versus the local mixity measure $\psi_{p}$.

Figure 18: Crack opening displacement at $45^{\circ}$ versus $\ell / R_{p}$ fro three different mode mixities $\psi_{p}$.

Figure 19: Mean stress, $\sigma_{m}$, at a distance equal to $0.1 \% R_{p}$ from the crack$\operatorname{tip}\left(\theta=0^{\circ}\right)$ as a function of the material length scales for various mode mixities $\psi_{p}$ 

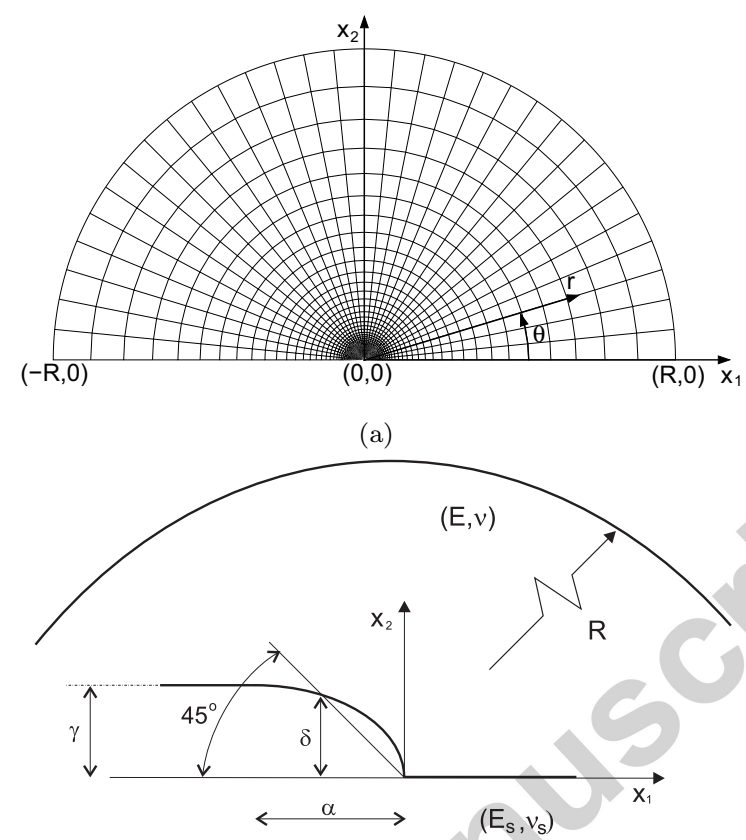

(b)

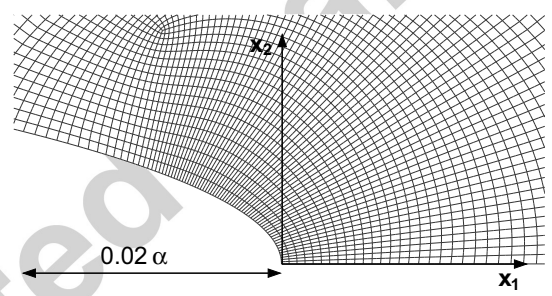

(c)

Figure 1: (a) Finite element mesh for the semi-infinite crack geometry , (b) schematic of the crack-tip geometry, and (c) near-tip mesh details. 


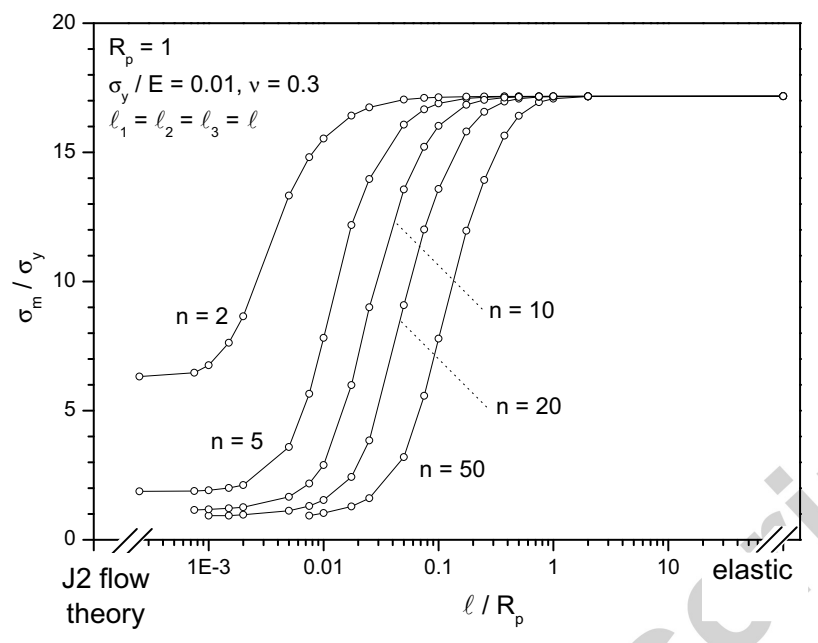

Figure 2: Mean stress at a distance equal to $0.1 \% R_{p}$ from the crack-tip $\left(\theta=0^{\circ}\right)$ as function of the material length scales for various strain hardening exponents.

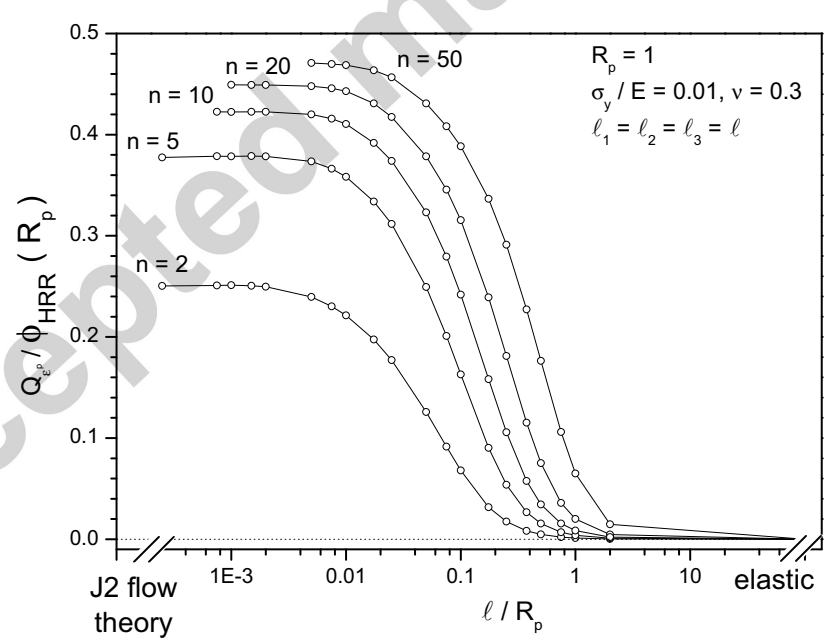

Figure 3: Normalised conventional plastic energy as function of the material length scales for various strain hardening exponents. 


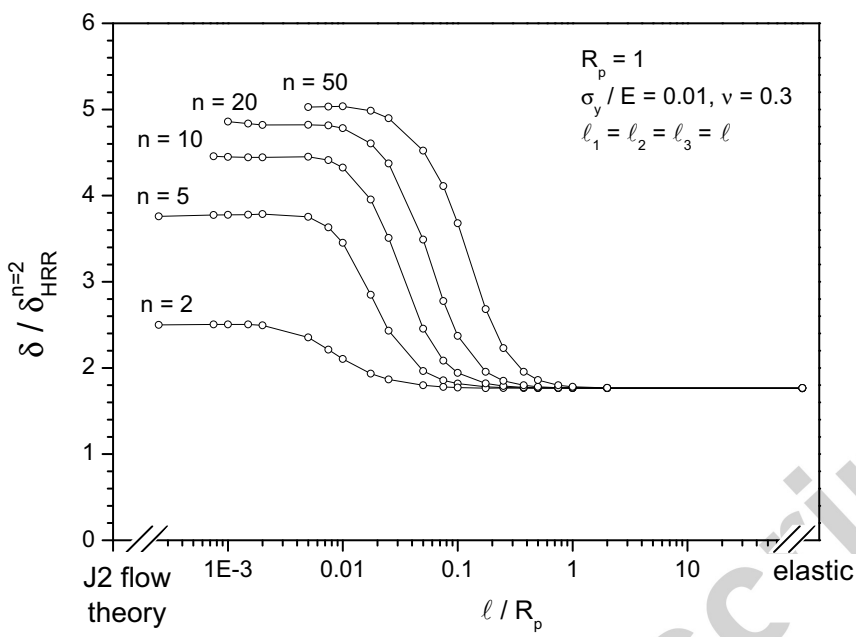

Figure 4: Normalised crack opening displacement as function of the material length scales for various strain hardening exponents.

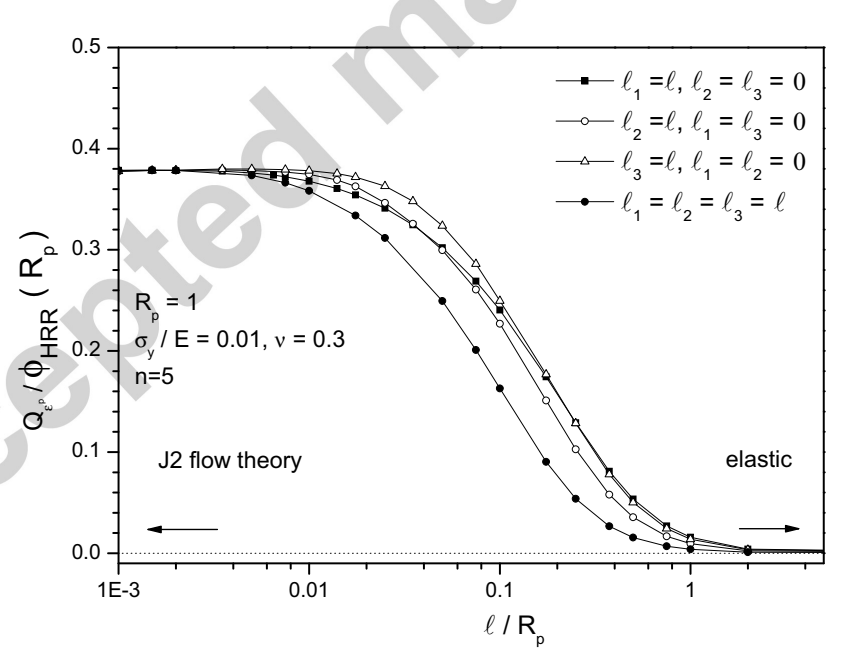

Figure 5: Normalised conventional plastic energy for various material length scales $(n=5)$. 


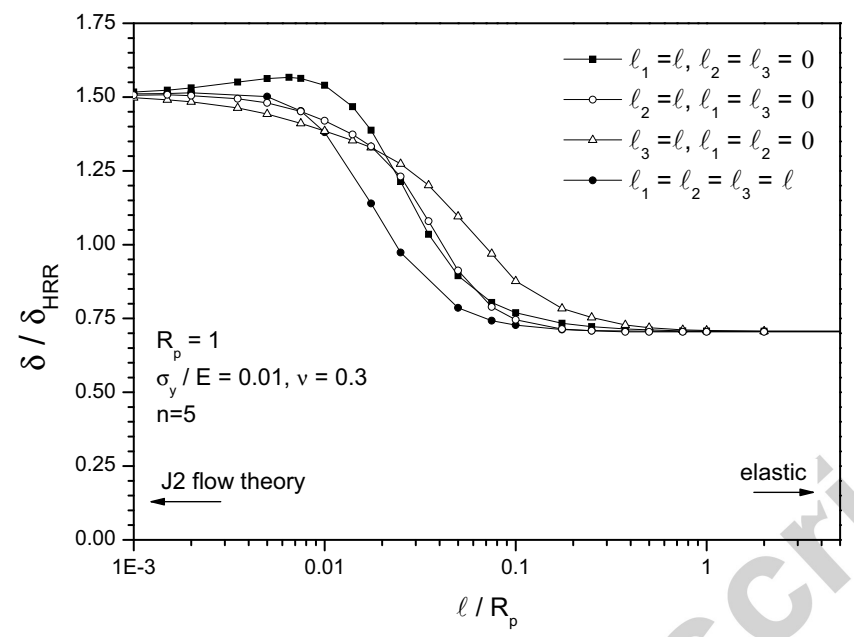

Figure 6: Normalised crack opening displacement for various material length scales $(n=5)$.

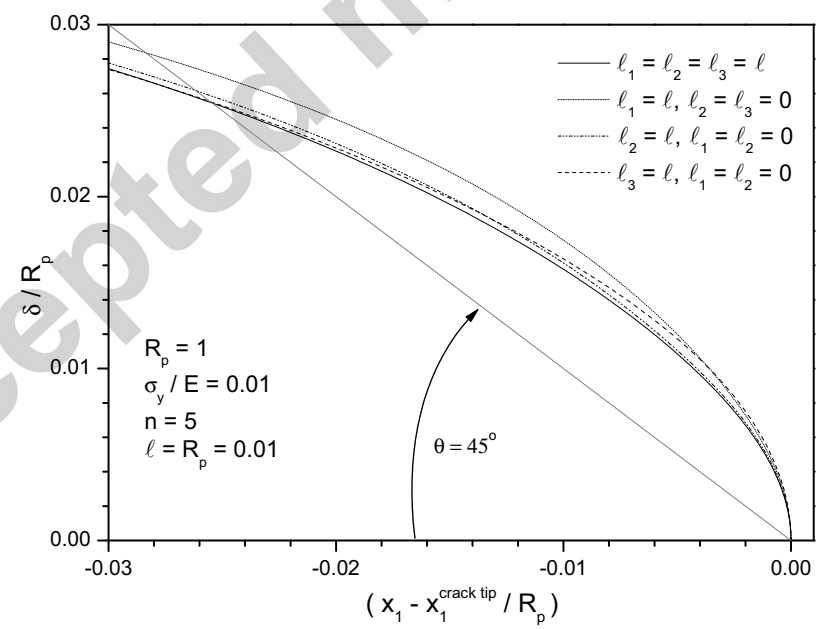

Figure 7: Crack opening profile $(n=5)$. 


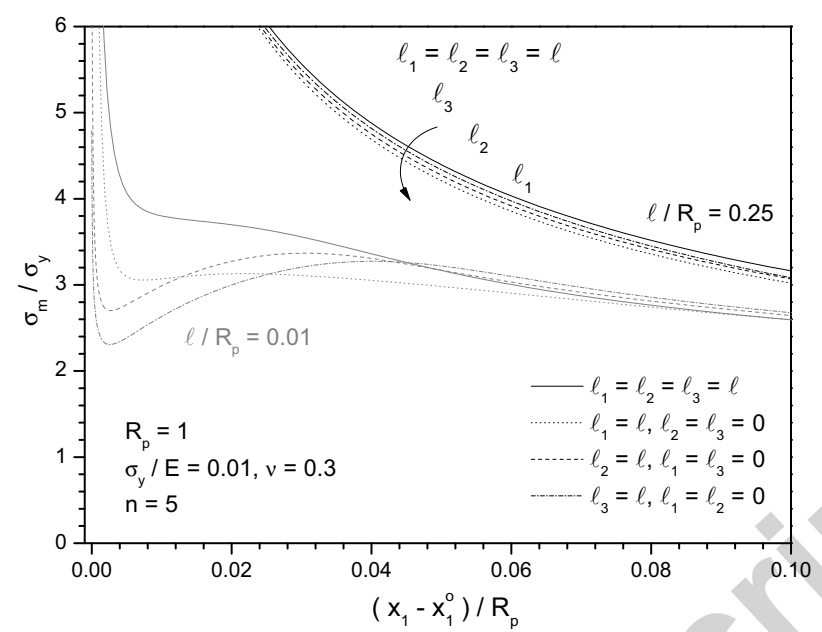

Figure 8: Mean stress, $\sigma_{m}$, along the symmetry line in front of the crack-tip.

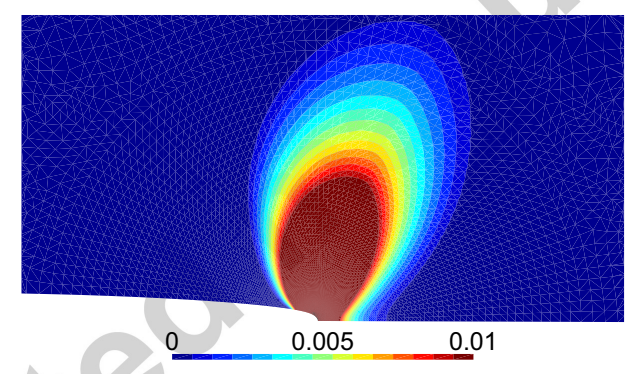

(a)

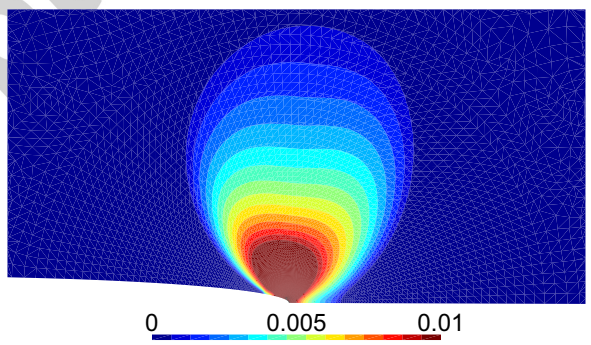

(b)

Figure 9: Contours of generalised effective plastic strain, $E^{p}$, for (a) $\ell / R_{p}=$ 0.005 , and (b) $\ell / R_{p}=0.25\left(\ell_{1}=\ell_{2}=\ell_{3}=\ell\right)$. 


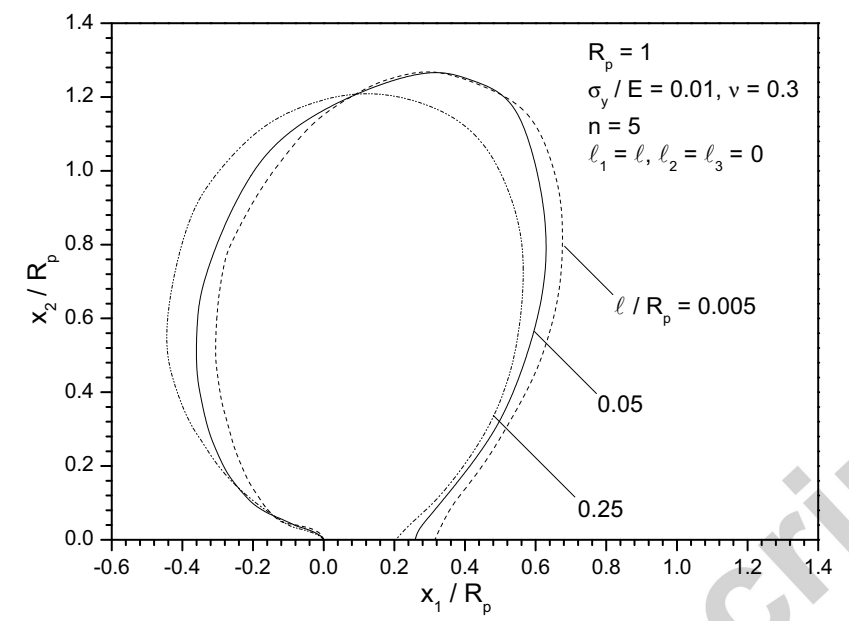

Figure 10: Small-scale yielding boundaries of the plastic zone for three different $\ell_{1}\left(\ell_{2}=\ell_{3}=0\right)$.

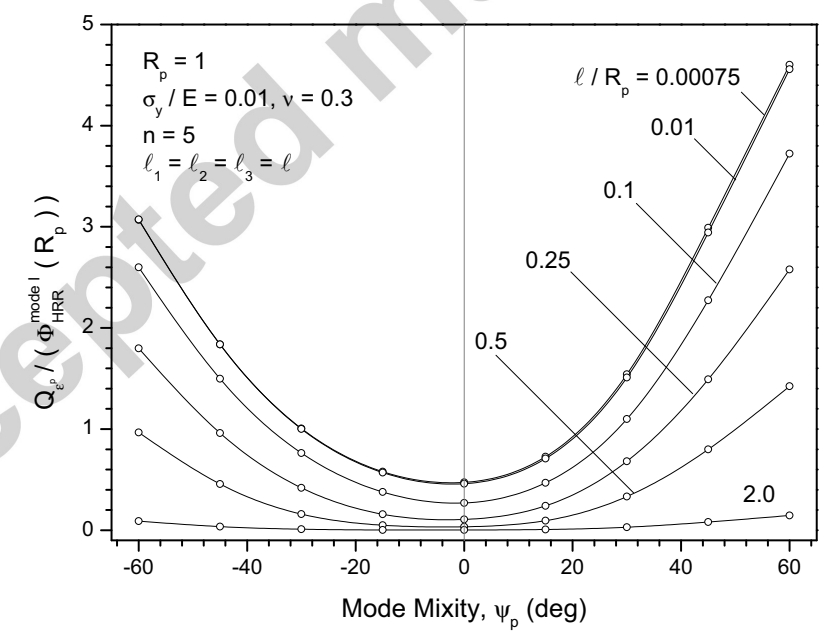

Figure 11: Crack tip normalised conventional plastic energy versus the local mixity measure $\psi_{p}$. 


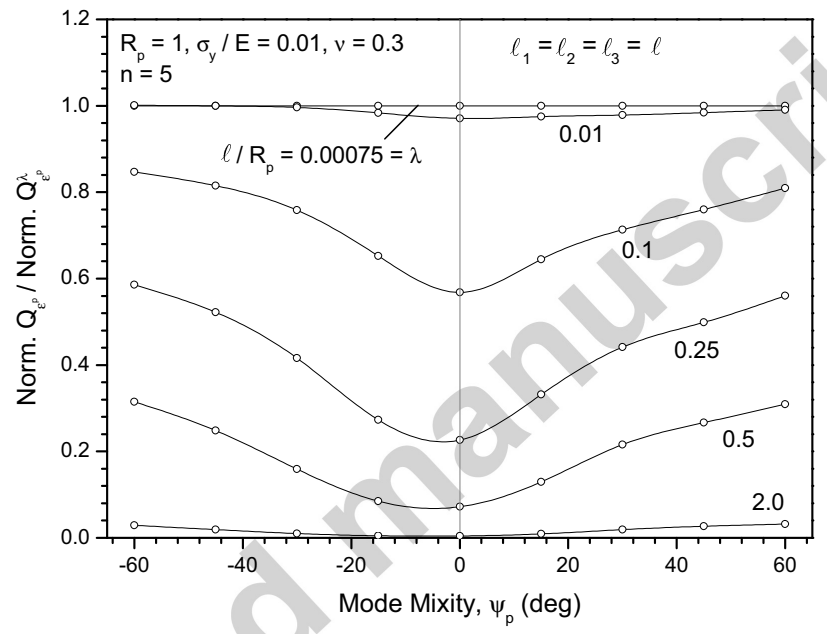

Figure 12: Normalised conventional plastic energy over the normalised $Q_{\varepsilon^{p}}$, for $\lambda=\ell / R_{p}=0.00075$, versus the local mixity measure $\psi_{p}$. The normalisation of $Q_{\varepsilon^{p}}$ is given in Fig. 11. 


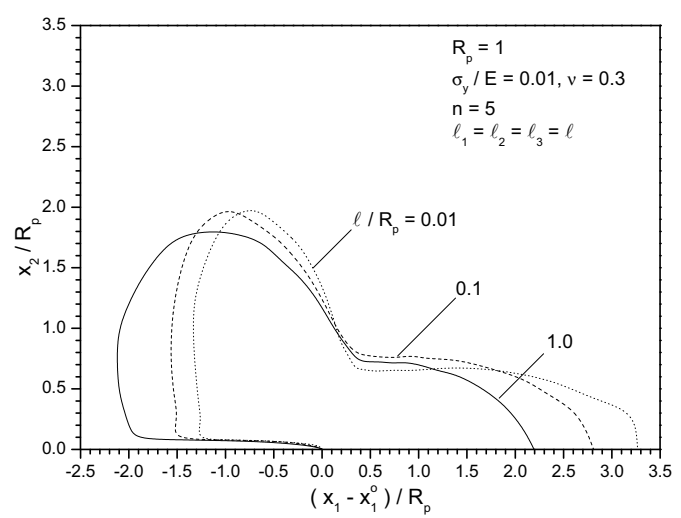

(a)

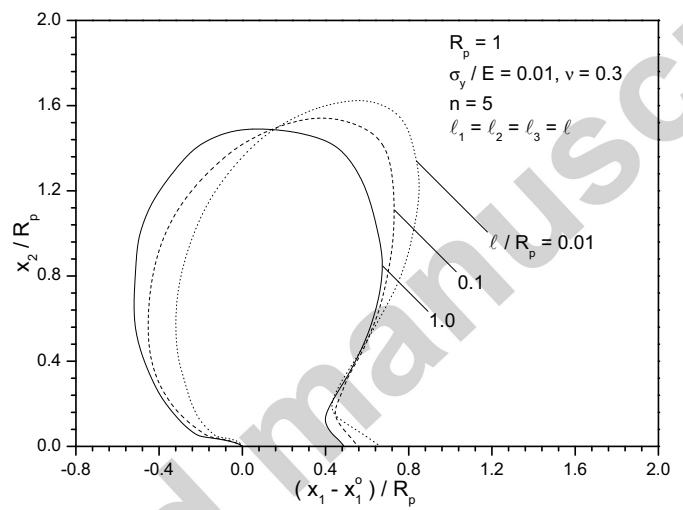

(b)

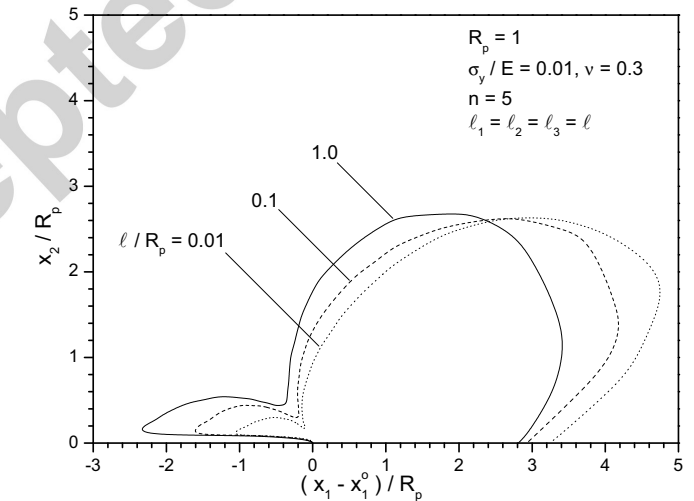

(c)

Figure 13: Boundaries of the plastic zone. The mode mixity is (a) $\psi_{p}=-45^{\circ}$, (b) $\psi_{p}=0^{\circ}$, and (c) $\psi_{p}=+45^{\circ}$. 


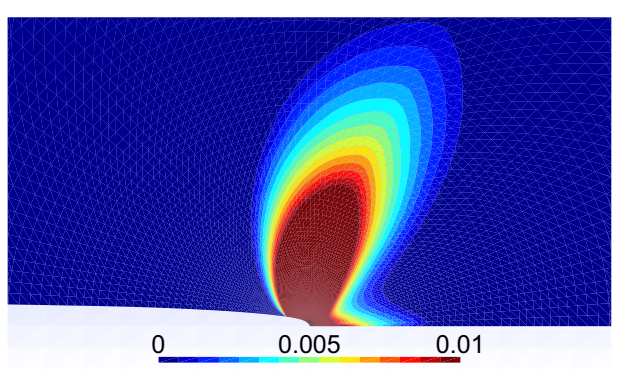

(a)

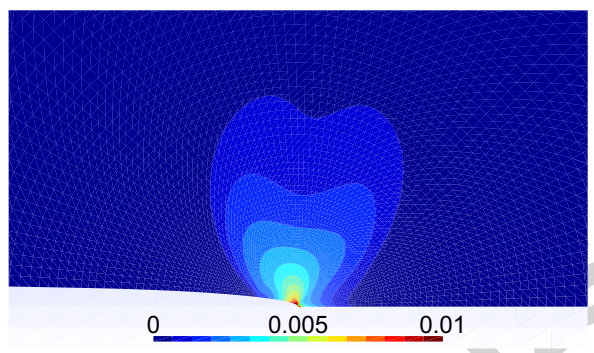

(b)

Figure 14: Contours of generalised effective plastic strain, $E^{p}$, for (a) $\ell / R_{p}=$ 0.01 , and (b) $\ell / R_{p}=1.0\left(\psi_{p}=0^{\circ}\right.$ and $\left.\ell_{1}=\ell_{2}=\ell_{3}=\ell\right)$. 


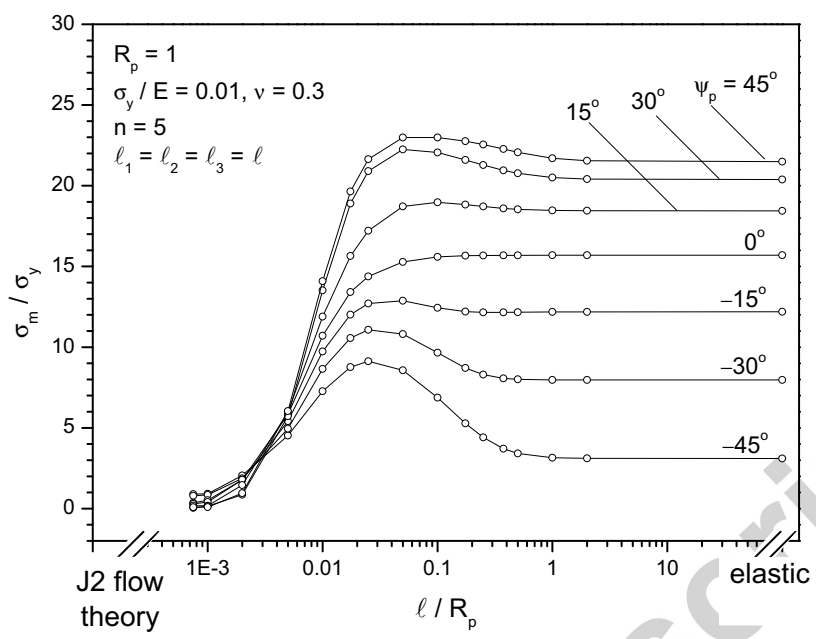

Figure 15: Mean stress at a distance equal to $0.1 \% R_{p}$ from the crack-tip $\left(\theta=0^{\circ}\right)$ as a function of the material length scales for various mode mixities $\psi_{p}$.

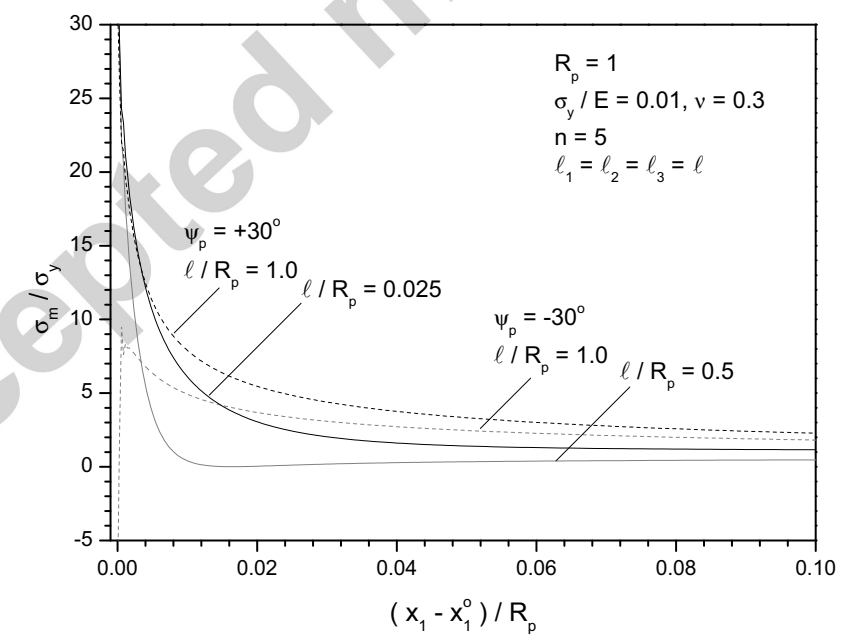

Figure 16: Mean stress, $\sigma_{m}$, along the interface in front of the crack-tip. 


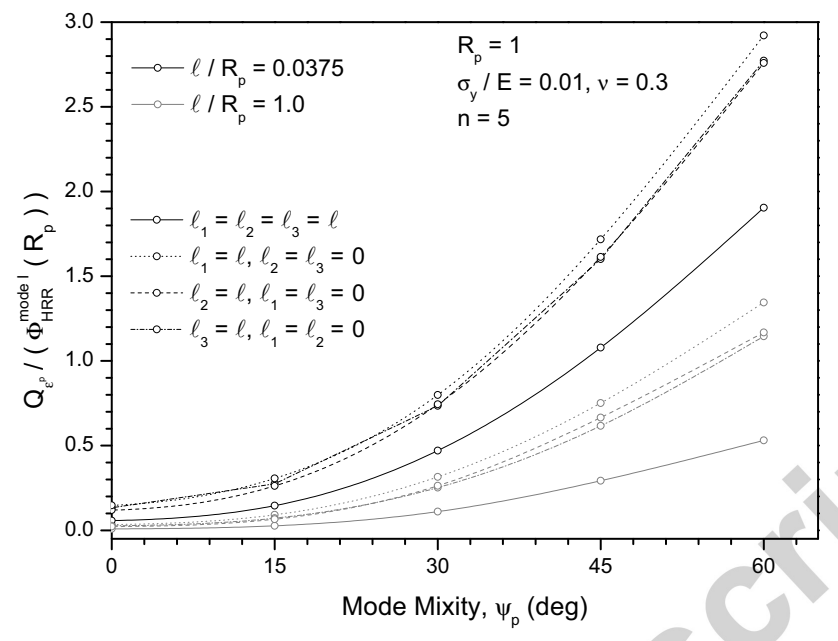

Figure 17: Crack tip normalised conventional plastic energy versus the local mixity measure $\psi_{p}$.

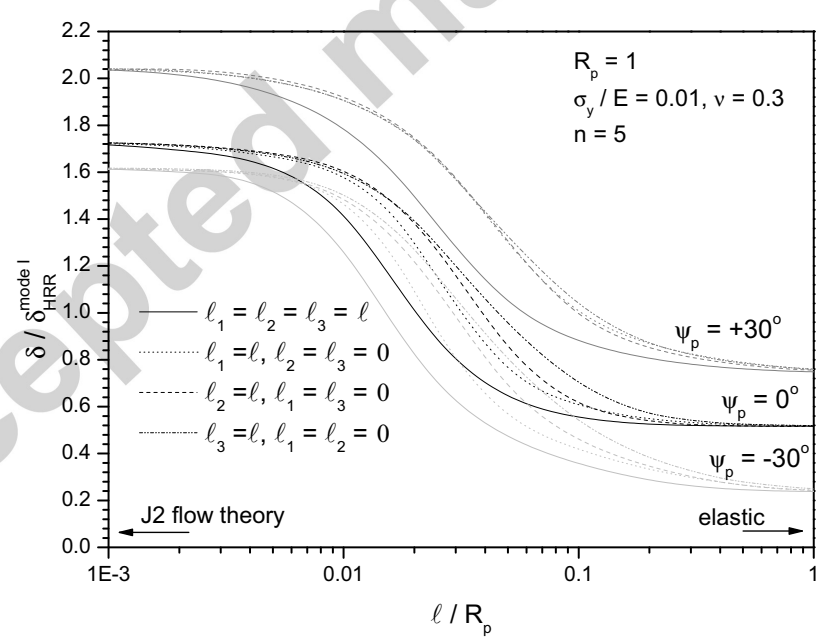

Figure 18: Crack opening displacement at $45^{\circ}$ versus $\ell / R_{p}$ fro three different mode mixities $\psi_{p}$. 


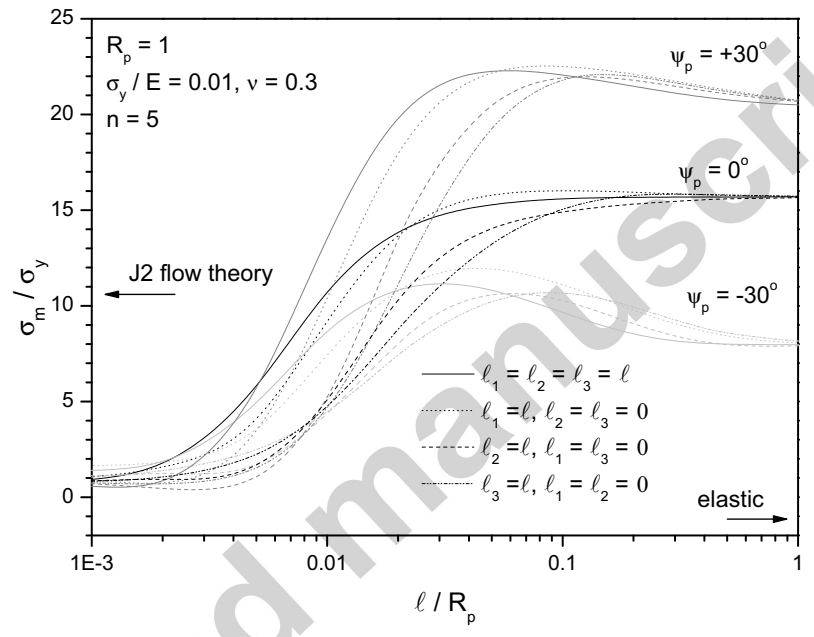

Figure 19: Mean stress, $\sigma_{m}$, at a distance equal to $0.1 \% R_{p}$ from the crack-tip $\left(\theta=0^{\circ}\right)$ as a function of the material length scales for various mode mixities $\psi_{p}$ 
- Investigation of Mode I and mixed mode cracks using strain gradient plasticity.

- A large strain finite element model (Hutchinson-Fleck theory) was developed.

- The length scale where a strain gradient plasticity is necessary was identified.

- The plastic zone size and shape dependence on the length scales was shown. 